\title{
Modeling Damage in Composite Materials Using an Enrichment Based Multiscale Method
}

\author{
Michael F. Macri \\ Andrew G. Littlefield
}

March 2015

ARMAMENT RESEARCH, DEVELOPMENT AND ENGINEERING CENTER Armaments Engineering \& Technology Center Weapon Systems \& Technology 
The views, opinions, and/or findings contained in this report are those of the author(s) and should not be construed as an official Department of the Army position, policy, or decision, unless so designated by other documentation.

The citation in this report of the names of commercial firms or commercially available products or services does not constitute official endorsement by or approval of the U.S. Government.

Destroy this report when no longer needed by any method that will prevent disclosure of its contents or reconstruction of the document. Do not return to the originator. 
Public reporting burden for this collection of information is estimated to average 1 hour per response, including the time for reviewing instructions, searching data sources,

gathering and maintaining the data needed, and completing and reviewing the collection of information. Send comments regarding this burden estimate or any other aspect of this collection

of information, including suggestions for reducing this burden to Washington Headquarters Service, Directorate for Information Operations and Reports,

1215 Jefferson Davis Highway, Suite 1204, Arlington, VA 22202-4302, and to the Office of Management and Budget,

Paperwork Reduction Project (0704-0188) Washington, DC 20503

PLEASE DO NOT RETURN YOUR FORM TO THE ABOVE ADDRESS.

1. REPORT DATE (DD-MM-YYYY)

02/03/2015

4. TITLEAND SUBTITLE

Modeling Damage in Composite Materials Using an Enrichment Based

Multiscale Method
3. DATES COVERED (From - TO)

\section{REPORT TYPE \\ Technical Report}

5a. CONTRACT NUMBER

5b. GRANT NUMBER

5c. PROGRAM ELEMENT NUMBER

6. AUTHOR(S)

Michael F. Macri

Andrew G. Littlefield

5d. PROJECT NUMBER

5e. TASK NUMBER

5f. WORK UNIT NUMBER

\section{PERFORMING ORGANIZATION NAME(S) AND ADDRESS(ES)}

U.S. Army ARDEC

Benet Laboratories, RDAR-WSB

Waterviet, NY 12189-4000

9. SPONSORING/MONITORING AGENCY NAME(S) AND ADDRESS(ES)

8. PERFORMING ORGANIZATION

REPORT NUMBER

ARWSB-TR-15002

12. DISTRIBUTION AVAILABILITYSTATEMENT

Approved for public release; distribution is unlimited.

\section{SUPPLEMENTARY NOTES}

\section{ABSTRACT}

Fielded and future military systems are increasingly incorporating composite materials into their design. Many of these systems subject the composites to physical trauma or environmental conditions that can cause microdamage leading to variations of the mechanical properties on the global scale. For these applications, it is critical to develop the ability to accurately model the response of composite materials, to enable engineers to predict the reaction of the system. To address this problem, a structural based enrichment approach is proposed, that allows macroscale computations to be performed with the microstructural features explicitly considered. This strategy has an advantage in that the enriched local function space may be easily varied from one element to the other allowing variances in the microstructure, such as localized damage to the fibers.

\section{SUBJECT TERMS}

Composite Materials; Structural Based Enrichment Approach; Enrichment Based Multiscale Method

\section{SECURITY CLASSIFICATIONOF:}

a. REPORT

$\mathrm{U} / \mathrm{U}$ b. ABSTRACT

U
17. LIMITATION OF ABSTRACT
18. NUMBER OF PAGES

15 19a. NAME OF RESPONSIBLE PERSON Michael Macri

19b. TELEPONE NUMBER (Include area code) (518) 266-5158 
1. REPORT DATE. Full publication date, including day, month, if available. Must cite at lest the year and be Year 2000 compliant, e.g., 30-06-1998; xx-08-1998; xx-xx-1998.

2. REPORT TYPE. State the type of report, such as final, technical, interim, memorandum, master's thesis, progress, quarterly, research, special, group study, etc.

3. DATES COVERED. Indicate the time during which the work was performed and the report was written, e.g., Jun 1997 - Jun 1998; 1-10 Jun 1996; May - Nov 1998; Nov 1998.

4. TITLE. Enter title and subtitle with volume number and part number, if applicable. On classified documents, enter the title classification in parentheses.

5a. CONTRACT NUMBER. Enter all contract numbers as they appear in the report, e.g. F33615-86-C-5169.

5b. GRANT NUMBER. Enter all grant numbers as they appear in the report, e.g. 1F665702D1257.

5c. PROGRAM ELEMENT NUMBER. Enter all program element numbers as they appear in the report, e.g. AFOSR-82-1234.

5d. PROJECT NUMBER. Enter al project numbers as they appear in the report, e.g. 1F665702D1257; ILIR.

5e. TASK NUMBER. Enter all task numbers as they appear in the report, e.g. 05; RF0330201; T4112.

5f. WORK UNIT NUMBER. Enter all work unit numbers as they appear in the report, e.g. 001; AFAPL30480105.

6. AUTHOR(S). Enter name(s) of person(s) responsible for writing the report, performing the research, or credited with the content of the report. The form of entry is the last name, first name, middle initial, and additional qualifiers separated by commas, e.g. Smith, Richard, Jr.

7. PERFORMING ORGANIZATION NAME(S) AND ADDRESS(ES). Self-explanatory.

\section{PERFORMING ORGANIZATION REPORT} NUMBER. Enter all unique alphanumeric report numbers assigned by the performing organization, e.g. BRL-1234; AFWL-TR-854017-Vol-21-PT-2.

\section{SPONSORING/MONITORS AGENCY} NAME(S) AND ADDRESS(ES). Enter the name and address of the organization(s) financially responsible for and monitoring the work.

10. SPONSOR/MONITOR'S ACRONYM(S). Enter, if available, e.g. BRL, ARDEC, NADC.

\section{SPONSOR/MONITOR'S REPORT}

NUMBER(S). Enter report number as assigned by the sponsoring/ monitoring agency, if available, e.g. BRL-TR-829; -215.

\section{DISTRIBUTION/AVAILABILITY}

STATEMENT. Use agency-mandated availability statements to indicate the public availability or distribution limitations of the report. If additional limitations/restrictions or special markings are indicated, follow agency authorization procedures, e.g. RD/FRD, PROPIN, ITAR, etc. Include copyright information.

13. SUPPLEMENTARY NOTES. Enter information not included elsewhere such as: prepared in cooperation with; translation of; report supersedes; old edition number, etc.

14. ABSTRACT. A brief (approximately 200 words) factual summary of the most significant information.

15. SUBJECT TERMS. Key words or phrases identifying major concepts in the report.

\section{SECURITY CLASSIFICATION. Enter} security classification in accordance with security classification regulations, e.g. $\mathrm{U}, \mathrm{C}, \mathrm{S}$, etc. If this form contains classified information, stamp classification level on the top and bottom of this page.

17. LIMITATION OF ABSTRACT. This block must be completed to assign a distribution limitation to the abstract. Enter UU (Unclassified Unlimited) or SAR (Same as Report). An entry in this block is necessary if the abstract is to be limited. 


\begin{abstract}
Fielded and future military systems are increasingly incorporating composite materials into their design. Many of these systems subject the composites to physical trauma or environmental conditions that can cause microdamage leading to variations of the mechanical properties on the global scale. For these applications, it is critical to develop the ability to accurately model the response of composite materials, to enable engineers to predict the reaction of the system. To address this problem, a structural based enrichment approach is proposed, that allows macroscale computations to be performed with the microstructural features explicitly considered. This strategy has an advantage in that the enriched local function space may be easily varied from one element to the other allowing variances in the microstructure, such as localized damage to the fibers.
\end{abstract}




\section{TABLE OF CONTENTS}

Abstract

\section{LIST OF FIGURES}

Figure 1: Multiscale Homogenization Approach ............................................................. 2

Figure 2: Implementation of Multiscale Enrichment Into FEA ….......................................... 7

Figure 3: Flow Chart of Structural Enrichment Approach...................................................... 7

Figure 4: Microstructures for a Fiber Composite............................................................. 8

Figure 5: Damaged Matrix Microstructure for a Fiber Composite ........................................... 8

Figure 6: Critical Region Around Global Crack .................................................................. 9

Figure 7: Critical Region Around Global Crack.................................................................. 9 


\section{LIST OF FIGURES (CONTINUED)}

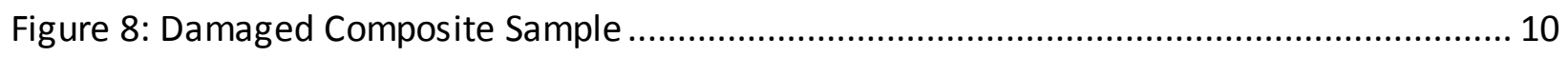

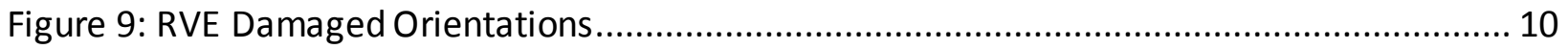

\section{LIST OF TABLES}

Table 1: Material Properties of RVE....................................................................................... 10

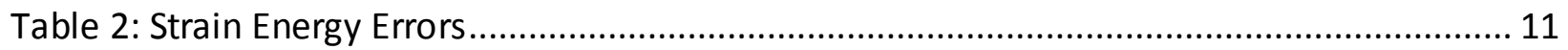

Table 3: Strain Energy Errors ....................................................................................................... 11 


\section{INTRODUCTION}

This presentation discusses the development of a technique to numerically model the reaction of composite materials that are undergoing microdamage. Damage on the microstructure of composites, such as microcracks and fracture, can have a significanteffect the gl obal response of the system. The most notable effect is in the change of material properties of the composite as microcracks form and propagate. Though empirical data can be used to approximate the material properties for an ideal non-damaged specimen [1], performing experimentation on damaged composites and extracting a correlation between microcrack size and or quantity to material properties can be extremely difficult.

One approach to extract material properties from a composite material without having to perform experimental testing is a numerical approximation method, called the homogenization method [2]. This method uses asymptotic expansions of field variables about macroscopic values and provides overall effective properties as well as microscopic stress and strain values. A limitation with this method, however, is that it assumes uniformity of the macroscopic fields within each representative volume element (RVE). Hence, this method breaks down in critical regions of high gradients such as cracks. This further complicates its use for modeling microcrack phenomena, as it is in the regions of global cracks that microcracking will be formed.

To overcome the drawbacks of the existing methods $[3,4]$ proposed a structural based enrichment method based on the principles of partition of unity which allows enrichment of the approximation space in localized sub domains using special ized functions that may be generated based on a priori information regarding asymptotic expansions of local stress fields and microstructure. One of the major advantages of the partition of unity-based enrichment strategies is that the enriched local function space may be easily varied from one node to the other.

In this presentation we review the development of RVE models which will correlate composite material properties, based on microcracking, and their incorporation into a modified structural based enrichment method to capture the microdamage effects. This approach can be used in regions where macrocracks are assumed to have initiated. In the next section, we review the homogenization and enrichment methods and discuss the implementation of damaged microstructures into the enrichment technique. In section 3, we present an example which demonstrates the effectiveness of our approach.

\section{EXPERIMENTATION}

We have divided this section into three parts. Section 2.1 and 2.2 review the concepts of the homogenization method and structural enrichment technique, respectively. Section 2.3 discusses the implementation of damaged microstructures into the structural enrichment technique. 


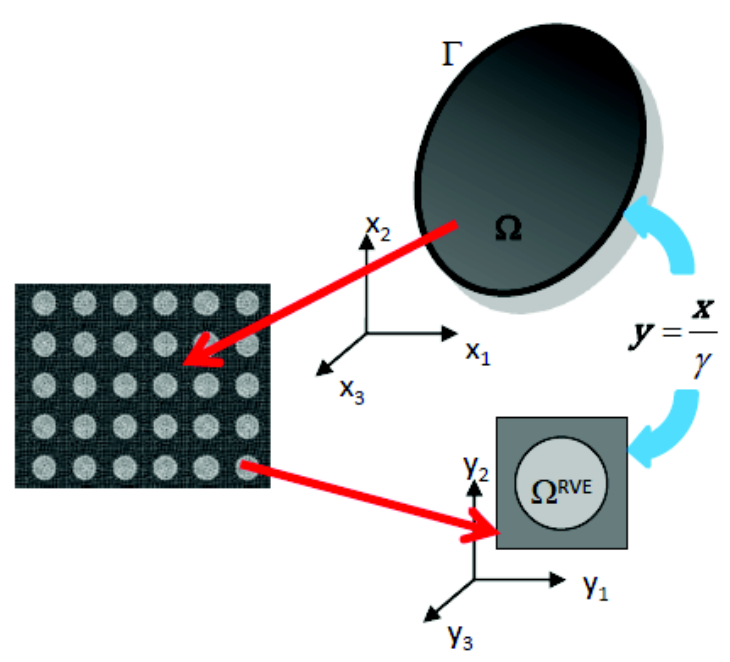

Figure 1. Multiscale Homogenization Approach

\section{Review of the Homogenization Approach}

The homogenization theory [2], assumes that a function, composed of multiple scales, can be designated as

$$
f^{\gamma}(x)=f(\boldsymbol{x}, \boldsymbol{y}(\boldsymbol{x}))
$$

where $\boldsymbol{x}$ and $\boldsymbol{y}$ represent a macroscale and microscale, respectfully (see Figure 1). The scales can be related by a scale factor, $\nu$, such that

$$
\boldsymbol{y}=\frac{\boldsymbol{x}}{\gamma}
$$

A key feature of the homogenization approach is the assumption of local-periodicity on the microscale. Thus, if we have a representative volume element (RVE), as shown in Figure 1, with a side length of $Y$ we can write

$$
f\left(x_{i}, y_{j}\right)=f\left(x_{i}, y_{j}+k Y_{j}\right)
$$

where $k$ is the periodic interval. The partial derivative for the function with respect to $x$ can be simplified to

$$
\partial_{x_{i}} f\left(x_{i}, y_{j}\right)=\partial_{x_{i}} f+\gamma^{-1} \partial_{y_{i}} f
$$

Using asymptotic expansion, we can write the displacement, stain and stress fields, with respect to the scale factor $\gamma$, as: 


$$
\begin{gathered}
u_{i}^{\gamma}(\boldsymbol{x}, \boldsymbol{y})=u_{i}^{0}(\boldsymbol{x}, \boldsymbol{y})+\gamma u_{i}^{1}(\boldsymbol{x}, \boldsymbol{y})+\mathcal{O}\left(\gamma^{2}\right) \\
\varepsilon_{i j}^{\gamma}(\boldsymbol{x}, \boldsymbol{y})=\gamma^{-1} \varepsilon_{i j}^{-1}(\boldsymbol{x}, \boldsymbol{y})+\varepsilon_{i}^{0}(\boldsymbol{x}, \boldsymbol{y})+\gamma \varepsilon_{i}^{1}(\boldsymbol{x}, \boldsymbol{y})+\mathcal{O}\left(\gamma^{2}\right) \\
\sigma_{i j}^{\gamma}(\boldsymbol{x}, \boldsymbol{y})=\gamma^{-1} \sigma_{i j}^{-1}(\boldsymbol{x}, \boldsymbol{y})+\sigma_{i}^{0}(\boldsymbol{x}, \boldsymbol{y})+\gamma \sigma_{i}^{1}(\boldsymbol{x}, \boldsymbol{y})+\mathcal{O}\left(\gamma^{2}\right)
\end{gathered}
$$

where

$$
\sigma_{i j}^{\gamma}=C_{i j k l} \varepsilon_{k l}^{\gamma}
$$

is the constitutive relationship for the constituents of the RVE. In this presentation we will assume that the constitutive behavior of each constituent of the RVE is linear elastic and known.

To determine the effects of the microstructure across the domain, we begin by substituting the asymptotic expansions into the equilibrium equation and rearranging the terms to give us:

$$
\begin{gathered}
\gamma^{-2}\left[\left(C_{i j k l} \varepsilon_{k l}^{-1}\right)_{, y_{j}}\right]+\gamma^{-1}\left[\left(C_{i j k l} \varepsilon_{k l}^{-1}\right)_{, x_{j}}+\left(C_{i j k l} \varepsilon_{k l}^{0}\right)_{y_{j}}\right] \\
+\gamma^{0}\left[\left(C_{i j k l} \varepsilon_{k l}^{0}\right)_{, x_{j}}+\left(C_{i j k l} \varepsilon_{k l}^{1}\right)_{, y_{j}}-f_{i}^{B}\right]+\mathcal{O}(\gamma)=0
\end{gathered}
$$

In equation [7], $C_{i j k l}$ is the elastic coefficients and $f_{i}^{B}$ is the body force. By setting each order term to zero we derive the following equations

$$
\begin{gathered}
u_{i}^{0}(\boldsymbol{x}, \boldsymbol{y})=u_{i}^{0}(\boldsymbol{x}) \\
{\left[C_{i j k l}\left(I_{k l m n}+\psi_{m n k l}^{S}\right)\right]_{, y_{i}}=0 \quad \epsilon \Omega^{\mathrm{RVE}}} \\
{\left[\tilde{C}_{i j k l} \varepsilon_{m n x}^{0}\right]_{, x_{j}}-\tilde{f}_{i}^{B}=0 \quad \epsilon \Omega}
\end{gathered}
$$

Equation [8] states that $u_{i}^{0}$ is only a function of the macroscale. Equation [9] is the governing equation for the microscale RVE, used to derive the effective elastic coefficients, where $\psi_{m n k l}^{S}$ is the symmetric part of the spatial gradient of $H_{m n k}^{S}$ defined as

$$
\psi_{m n k l}^{S}=\frac{1}{2}\left(H_{m n l, y_{k}}^{S}+H_{m n k, y_{l}}^{S}\right)
$$

$\boldsymbol{H}^{5}(\boldsymbol{y})$ is a Y-periodic tensor that relates the macroscale to the first order displacement perturbation term $u_{i}^{1}(\boldsymbol{x}, \boldsymbol{y})$, such that:

$$
u_{i}^{1}(\boldsymbol{x}, \boldsymbol{y})=H_{m n i}^{S}(\boldsymbol{y}) \varepsilon_{m n x}^{0}(\boldsymbol{x})
$$

Equation [10] is the governing equation for the macroscale. In the equation, $\widetilde{\boldsymbol{C}}$ represents the effective elastic coefficients and $\tilde{\boldsymbol{f}}^{B}$ is the effective body force. They are respectively defined as: 


$$
\begin{gathered}
\tilde{C}_{i j k l}=\frac{1}{V^{R V E}} \int_{\Omega^{\mathrm{RVE}}} \mathrm{C}_{i j k l}\left(I_{k l m n}+\Psi_{\mathrm{mnkl}}^{\mathrm{S}}\right) \partial \Omega^{\mathrm{RVE}} \\
\tilde{\mathrm{f}}_{\mathrm{i}}^{\mathrm{B}}=\frac{1}{V^{R V E}} \int_{\Omega^{\mathrm{RVE}}} \mathrm{f}_{\mathrm{i}}^{\mathrm{B}} \partial \Omega^{\mathrm{RVE}}
\end{gathered}
$$

\section{Structural Enrichment Method}

Within this section we review the partition of unity paradigm and how it is implemented, followed by the derivation of the enrichment functions.

\section{Interpolation Functions}

The partition of unity paradigm requires that the sum of all functions at a given point in a domain is equal to one, such that

$$
\sum_{i=1}^{N} \varphi_{I}^{0}(\boldsymbol{x})=1
$$

In the above equation, $\varphi_{I}^{0}$ is the $I^{\text {th }}$ partition of unity function and $N$ is the total number of partition of unity functions within the domain, $\Omega$. Thus, the sum of all the functions at a given point, $\boldsymbol{x}$, within $\Omega$ will equal 1.

Based on this concept we define the global approximation space, for a method using interpolation functions, as:

$$
V^{h, p}=\sum_{I=1}^{N} \varphi_{I}^{0} V_{I}^{h, p} \subset H^{1}(\Omega)
$$

where the superscripts $h$ and $p$ are the size of the element and the polynomial order, respectively. $H^{1}$ is the first order Hilbert space and $V_{I}^{h, p}$ is the local approximation space at $I$, defined as:

$$
V_{I}^{h, p}=\operatorname{span}_{m \in \zeta}\left\{p_{m}(\boldsymbol{x})\right\} \subset H^{1}\left(\bar{B}_{I} \cap \Omega\right)
$$

In equation [17], $\zeta$ is an index set, $\bar{B}_{I}$ is the support for node $I$ and $p_{m}(\boldsymbol{x})$ is composed of polynomials or otherfunctions. From the above equations we can write any function $v^{h, p}$ within the global approximation space as:

$$
v^{h, p}(x)=\sum_{I=1}^{N} \sum_{m \in \zeta} h_{I m}(\boldsymbol{x}) \alpha_{I m}, \quad v^{h, p} \in V^{h, p}
$$

where

$$
h_{I m}(\boldsymbol{x})=\varphi_{I}^{0}(\boldsymbol{x}) p_{m}(\boldsymbol{x})
$$

is the interpolation function at node $I$, corresponding to the $m^{\text {th }}$ degree of freedom, and $\alpha_{I m}$ is the associated degree of freedom. 
For FEA, the standard shape function, $N_{l}$, which can be referenced in numerous sources, satisfies the partition of unity criteria for $\varphi_{I}^{0}$. For a typical finite element analysis, correlating the definition of a finite element shape function to the interpolation function in equation [19], requires that $p_{m}=\operatorname{span}\{1\}$. Reference [5] discusses other partition of unity functions and basis's that can be implemented.

\section{Enrichment Functions}

In the previous section we defineda function in the global approximation space as the sum of the partition of unity functions multiplied by a group of basis functions and associated degrees of freedom. Focusing on the basis functions, $p_{n}(x)$, we state that if an arbitrary function is included in the local basis of all nodes within the domain and assuming $\alpha_{I m}=\delta_{m n} \forall I$ then

$$
\sum_{I=1}^{N} \sum_{m \in \zeta} \phi_{I}^{0}(\boldsymbol{x}) p_{m}(\boldsymbol{x}) \alpha_{I m}=p_{n}(x)\left(\sum_{I=1}^{N} \phi_{I}^{0}(\boldsymbol{x})\right)=p_{n}(\boldsymbol{x})
$$

Equation [20] demonstrates that it is possible to exactly reproduce a function over the entire domain. This fundamental concept enables the consistency of the interpolation functions to be adjusted based on the population of the basis functions. Thus, we can expend the basis function to include relevant functions to enforce a known displacement field. Recalling that the basis for a standard FEA analysis is $p_{m}=\operatorname{span}\{1\}$, and incorporating the asymptotic response about a crack tip, a common enrichment technique is to adjust the basis to

$$
p_{m}(\boldsymbol{x})=\operatorname{span}\{1, \sqrt{r}\}
$$

Using this adjusted basis within the region of a crack tip will enable the interpolation functions to reproduce the $\sqrt{r}$ field. If the crack tip is not at the origin of $r$ the degree of freedom associated with the enrichment function should solve to zero, thus removing the effects of the enrichment term on the displacement field.

To derive the structural based enrichment function, we begin by examining the asymptotic expansion of the displacement field given in equation [5a]. Substituting equations [8] and [12] into [5a] and assuming $\mathcal{O}\left(\gamma^{2}\right)$ approximation gives us:

$$
u_{i}^{\gamma}(\boldsymbol{x}, \boldsymbol{y})=u_{i}^{0}(\boldsymbol{x})+\gamma\left(H_{m n i}^{S}(\boldsymbol{y}) \varepsilon_{m n x}^{0}(\boldsymbol{x})\right)
$$

For a single-scale problem, we define the displacement field, assuming linear consistency in 2D, as

$$
u_{i}^{\gamma}(\boldsymbol{x})=u_{i}^{0}(\boldsymbol{x})=\sum_{I=1}^{N} \sum_{m \in \zeta} \phi_{I}^{0}(\boldsymbol{x}) p_{m}(\boldsymbol{x}) \alpha_{I m i}
$$

where $\varphi_{I}^{0}$ and $p_{m}$ are $N_{l}$ and $p_{m}=\operatorname{span}\{1\}$.

To perform a multiscale analysis we need to incorporate the second term on the right hand side of equation [22]. We focus on the Y-periodic tensor, $\boldsymbol{H}^{5}(\boldsymbol{y})$. Recalling that $\boldsymbol{y}$ is a function of $\boldsymbol{x}$, equation [2] and assuming $\boldsymbol{H}^{S}(\boldsymbol{y}(\boldsymbol{x}))$ is the dominant term, we can write the relation: 


$$
\gamma\left(H_{m n i}^{S}(\boldsymbol{y}) \varepsilon_{m n x}^{0}(\boldsymbol{x})\right)=\sum_{I=1}^{N} \phi_{I}^{0}(\boldsymbol{x}) H_{m n i}^{S}(\boldsymbol{y}(\boldsymbol{x})) \beta_{I m n i}
$$

Substituting [23] and [24] into [22] gives us:

$$
u_{i}^{\gamma}(\boldsymbol{x})=\sum_{I=1}^{N} \sum_{m \epsilon \zeta} \phi_{I}^{0}(\boldsymbol{x}) p_{m}(\boldsymbol{x}) \alpha_{I m i}+\sum_{I=1}^{N} \phi_{I}^{0}(\boldsymbol{x}) H_{m n i}^{S}(\boldsymbol{y}(\boldsymbol{x})) \beta_{I m n i}
$$

Rearranging [25] and incorporating the degrees of freedom vector $\boldsymbol{B}$ into $\boldsymbol{\alpha}$ reduces [25] to

$$
u_{i}^{\gamma}(\boldsymbol{x})=\sum_{I=1}^{N} \sum_{m \in \zeta} \phi_{I}^{0}(\boldsymbol{x}) \boldsymbol{p}_{m i}(\boldsymbol{x}) \boldsymbol{\alpha}_{I m i}
$$

The enrichment function can be defined as:

$$
\mathcal{F}_{m n i}^{1}(\boldsymbol{x})=H_{m n i}^{S}(\boldsymbol{y}(\boldsymbol{x}))
$$

One significant observation is that incorporating the enrichment function requires that the basis be dependent on the coordinate direction. For example, a two dimensional finite element analysis would have the enriched basis in the $x_{1}$-direction, as

$$
p_{m 1}=\operatorname{span}\left\{1, H_{111}^{S}(\boldsymbol{y}(\boldsymbol{x})), H_{221}^{S}(\boldsymbol{y}(\boldsymbol{x})), H_{121}^{S}(\boldsymbol{y}(\boldsymbol{x}))\right\}
$$

while for the $x_{2}$-direction the enriched basis is

$$
p_{m 2}=\operatorname{span}\left\{1, H_{112}^{S}(\boldsymbol{y}(\boldsymbol{x})), H_{222}^{S}(\boldsymbol{y}(\boldsymbol{x})), H_{122}^{S}(\boldsymbol{y}(\boldsymbol{x}))\right\}
$$

In generic form for a finite element simulation, the basis can be written as

$$
p_{m i}=\operatorname{span}\left\{1, \mathcal{F}_{m n i}^{1}(\boldsymbol{x})\right\}
$$

\section{Implementation the Multiscale Enrichment Technique}

The approach to implementing structural based enrichment varies depending on the governing method. In this presentation we will focus in the FEA approach. Reference [3] gives complete details on the implementation of the multiscale enrichment into FEA. In this section we will briefly review how the algorithm is applied.

The analysis is based on a homogenization-like integration (HLI) scheme [3]. The sol'n requires the enrichment function, calculated using equation [9], and the material properties, which are only known on the microscale, $\boldsymbol{y}$. To implement this technique and resolve the aforementioned issue, integration will be performed on an RVE transformed to the macroscale, $\boldsymbol{x}$, coordinate system. The HLI scheme, shown in Figure 2, is based on the assumption that there is a significant deference between the two scalesand that the Gauss points used for FEA are sparse with respect to the size of the RVE. In this approach, an RVE is centered on each Gauss point within macroscale problem. An integrand, I, on the macroscale is replaced with the following 


$$
I=\int_{\Omega} J v \partial \Omega=\sum_{i=1}^{n_{\text {guass }}} W_{i} J_{i} v\left(\boldsymbol{x}_{i}^{G P}\right)=\sum_{i=1}^{n_{\text {guass }}} W_{i} J_{i} \frac{1}{V^{R V E}} \int_{\bar{\Omega}_{\mathrm{i}}^{\mathrm{RVE}}} v(\boldsymbol{x}) \partial \bar{\Omega}_{\mathrm{i}}^{\mathrm{RVE}}
$$

Where $v$ is an arbitrary function, $x_{i}^{G P}$ is the $i^{\text {th }}$ Gauss point, $\bar{\Omega}_{i}^{R V E}$ is the domain of an RVE centered on the $i^{\text {th }}$ Gauss point, $J$ is the Jacobian and $W_{i}$ is a weight associate $d$ with the $i^{\text {th }}$ Gauss point. [3] has demonstrated that the accuracy of HLI improves with decreasing size of the RVE. Thus, this implementation works well when the difference in scales is significant. However, as the scales become closer and the therefore the volume of the RVE increases, equation [31] will develop convergence errors.

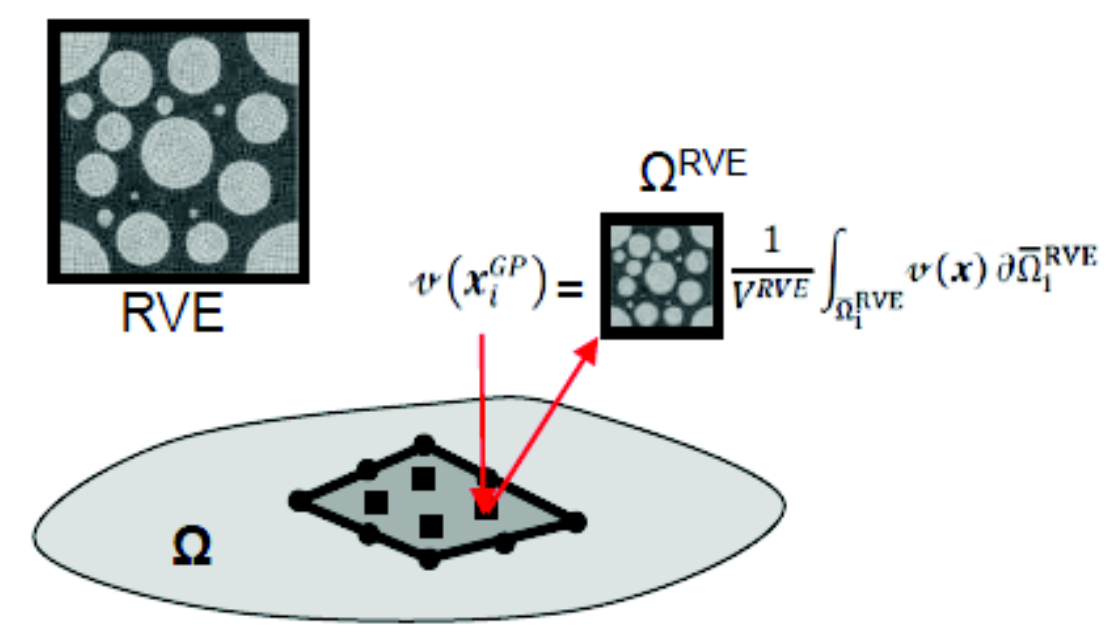

Figure 2. Implementation of multiscale enrichment into FEA

\section{Flow Chart of Structural Based Ennichment Algorithm}

In this section, we discuss the algorithm for the structural based enrichment method depicted in Figure 3. We begin the analysis by deriving the $\boldsymbol{H}^{S}$ from the RVE and material properties provided. $\boldsymbol{H}^{S}$ is then inputted into the macroscale structural problem which solves for the displacement, stress and strain fields.

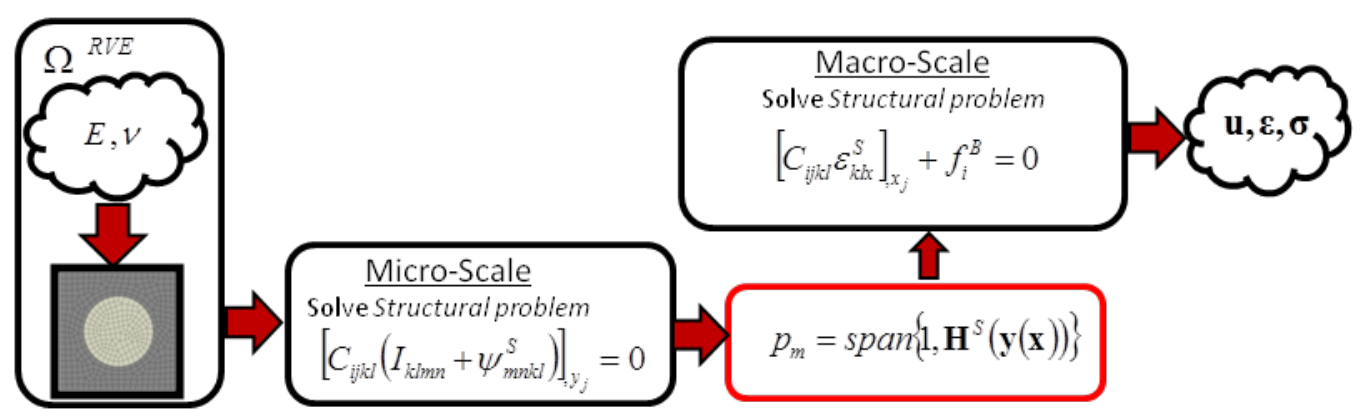

Figure 3. Flow Chart of Structural Enrichment Approach 


\section{Implementation of Damaged Microstructure}

We now focus, in this section, on the RVE and how microdamage can be incorporated into the model. For many applications the material used in the multiscale model is some type of fiber composite, such that the microstructure can be presented as a fiber within a matrix (see Figure 4a) for a 2D application, and a section of the fiber weave (see Figure $4 b$ ) for 3D simulations.

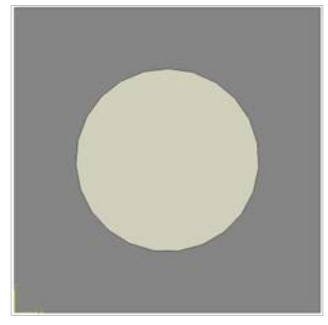

(a)

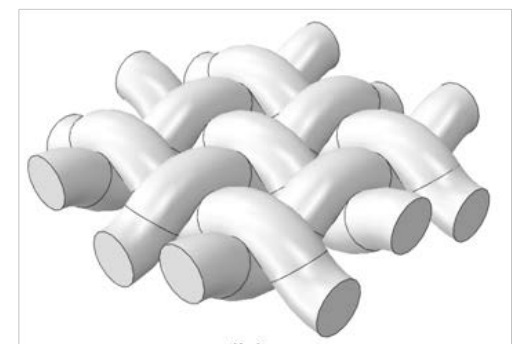

(b)

Figure 4. Microstructures for a Fiber Composite

For normal conditions, these RVEs can be used to represent the microstructure; however, these multiscale methods are specifically designed for critical regions, such as in the vicinity of a global crack, where fibermatrix is likely to be damaged on the microscale as well.
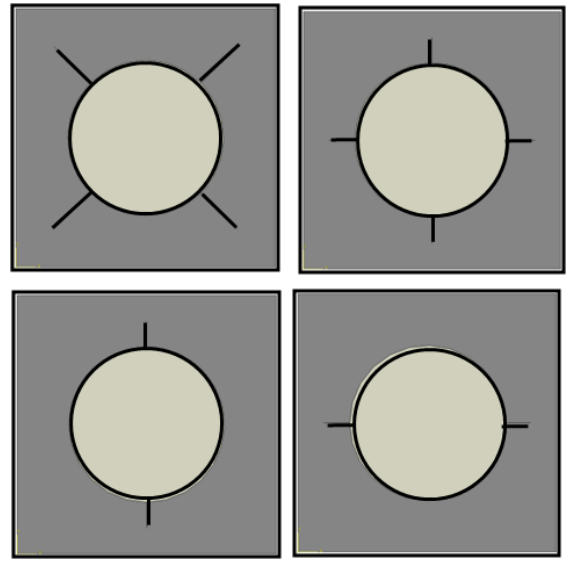

Figure 5. Damaged Matrix Microstructures for a Fiber Composite

To account for microdamage, the RVEs are modified to incorporate varying level sof cracks. Figure 5 gives examples of several cases where cracks are imbedded into the microstructure matrix.

The cracks on the RVE are modeled as geometric damage in the simulation, such that there is no connectivity across a crack on elements within the RVE neighboring the crack. As a pre-step to solving the global problem, we solve equation [9] on the damaged RVE to produce a set of damaged enrichment functions, $H_{m n i}^{S-D}$. The damaged enrichment functions are then incorporated into model within the vicinity of a global crack, see Figure 6. 


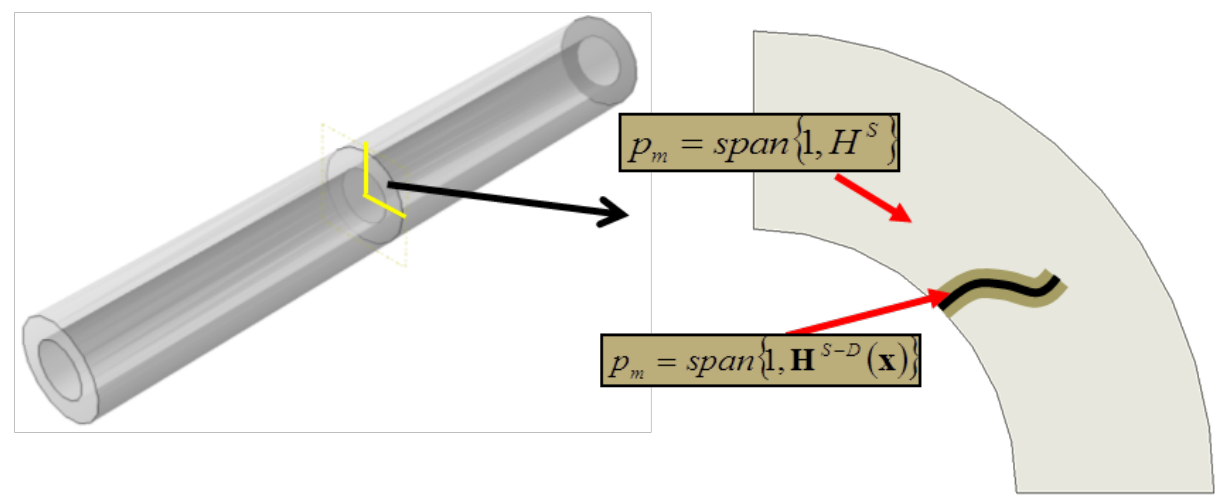

Figure 6. Critical Region Around Global Crack

The algorithm for the modified enrichment method is given pictorially in Figure 7 . We begin the analysis by deriving the $\boldsymbol{H}^{S}$ and $\boldsymbol{H}^{S-D}$ from the RVE and material properties provided. $\boldsymbol{H}^{S}$ and $\boldsymbol{H}^{S-D}$ are then inputted into the macroscale structural problem, in the form of enrichment functions. The enrichment functions derived from the damaged RVE, $\boldsymbol{H}^{S-D}$, are applied to elements within the vicinity of the critical region, while the remaining elements within the global model are enriched with functions derived from the standard RVE, $\boldsymbol{H}^{S}$. The profile of the critical region is dependent on criteria provided by the modeler.

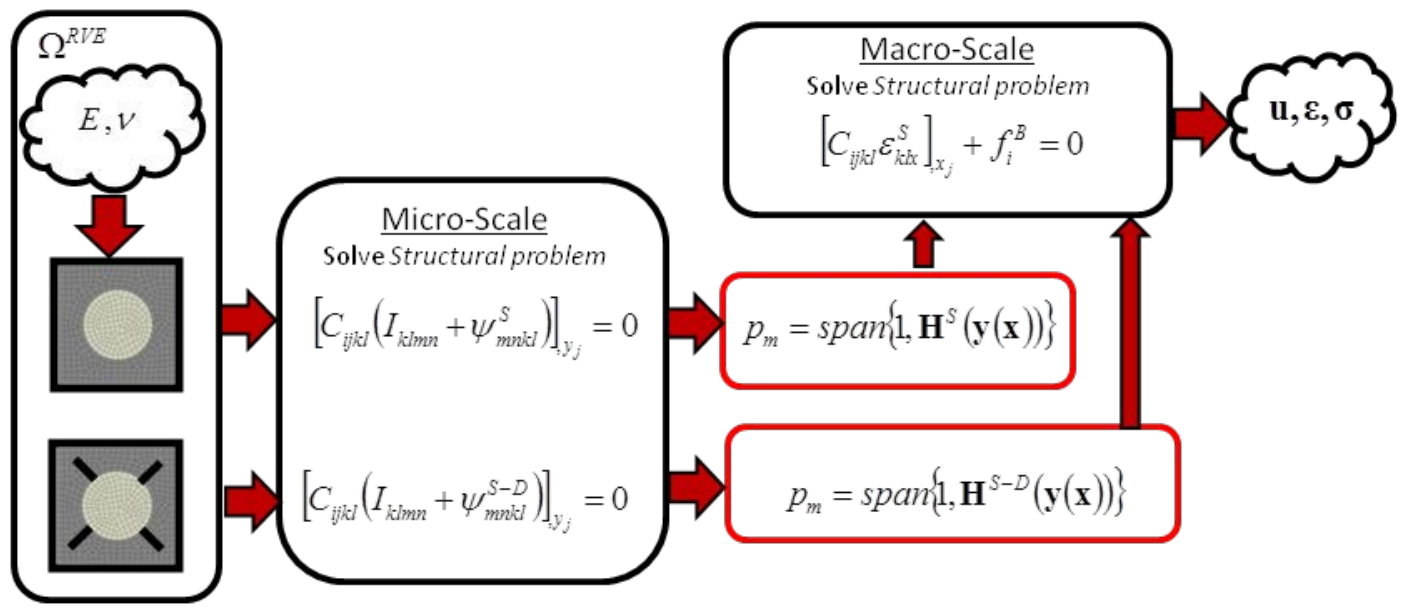

Figure 7. Critical Region Around Global Crack

\section{RESULTS}

We examine a two-dimensional cross section of a damaged composite wafer, depicted in Figure 8, containing a center crack. A pressure load is applied to the upper and lower surfaces to force open the crack and a temperature change is implemented to induce thermal stress. The additional enrichment functions used to capture the thermal stress is reviewed in [5]. In this example we assume plane stress conditions and examine the upper right quadrant of the cross sectional area. We assume the composite is composed of graphite fibers, all orientated perpendicular to the cross section, within an epoxy matrix. The material properties are given in Table 1 . The RVEs, are modeled using 500 quadratic $2^{\text {nd }}$ order elements, where the enrichment functions generated from the damaged RVE's are applied to the global region encompassed by the yellow boundary and the enrichment function generated from the standard 
RVE is applied throughout the remainder of the model. The boundary conditions on the global model are a $40 \mathrm{MPa}$ pressure pulling on the upper and lower surfaces of the wafer, and a heat source increasing the upper and lower surfaces to $1080^{\circ} \mathrm{C}$. The results are compared to a fine mesh composed of $1.8 \mathrm{e} 6$ quadratic $2^{\text {nd }}$ order elements modeled down to the microscale.

Table 1. Material Properties of RVE

\begin{tabular}{|l|l|l|l|l|}
\hline Material & $\begin{array}{l}\text { Elastic Modulus } \\
(\mathrm{MPa})\end{array}$ & $\begin{array}{l}\text { Poisson's } \\
\text { Ratio }\end{array}$ & $\begin{array}{l}\text { Thermal Conductivity } \\
(\mathrm{mW} /(\mathrm{mmC}))\end{array}$ & $\begin{array}{l}\text { Coefficient of Thermal } \\
\text { Expansion }\left(\mathrm{C}^{-1}\right)\end{array}$ \\
\hline Epoxy & 3450 & 0.35 & 4.47 & $5.4 \times 10^{-5}$ \\
\hline Graphite & 700 & 0.485 & 2.4 & $1.2 \times 10^{-5}$ \\
\hline
\end{tabular}

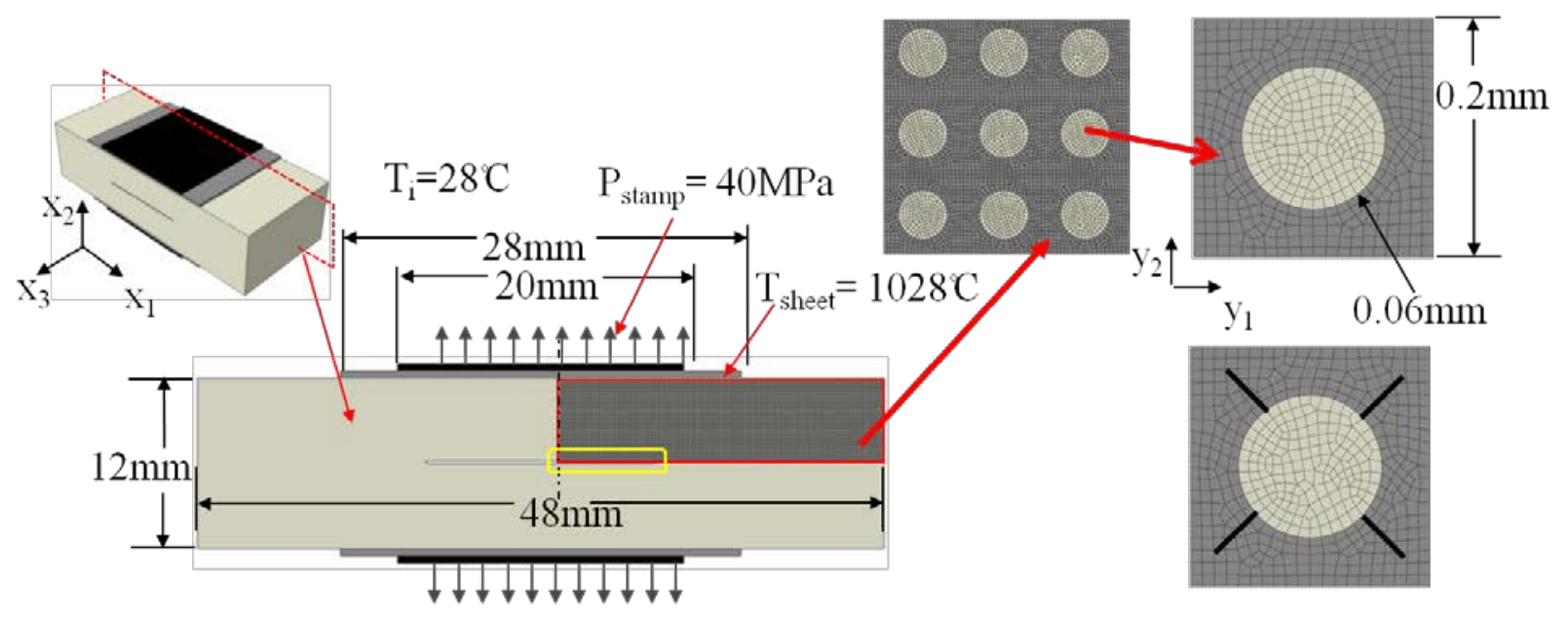

Figure 8. Damaged Composite Sample

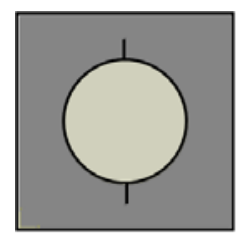

(a)

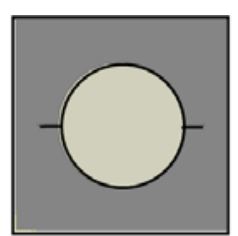

(b)

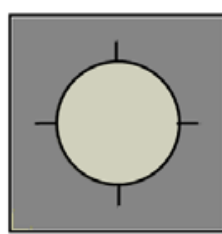

(c)

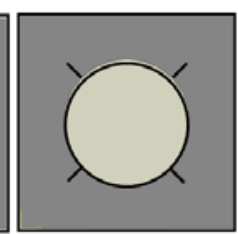

(d)

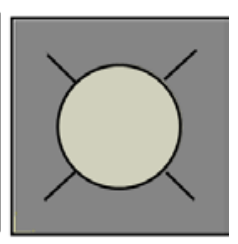

(e)

Figure 9. RVE Damaged Orientations

We begin the analysis by examining several crack orientations shown in Figure 9 . For this analysis, the homogenization simulation is run using 900 quadratic $2^{\text {nd }}$ order elements, with the effective material properties generated from a damaged RVE applied to the critical region around the crack. To compare the orientations, we examine the strain energy resulting from the simulation with respect to the results from the fine mesh which produced a strain energy of $882.4 \mathrm{MPa}$. Table 2 shows the resulting relative error in 
the strain energies. The results show that the orientation does not make significant difference in error generated. The RVE containing diagonal cracks with twice the crack length as the other orientations (Figure 9e) produced slightly more accurate results.

Table 2. Strain Energy Errors

\begin{tabular}{|c|c|c|}
\hline Technique & Strain Energy (MPa) & Error (\%) \\
\hline Fine Mesh & 882.4 & - \\
\hline Figure 9a & 213.2 & 75.8 \\
\hline Figure 9b & 213.3 & 75.8 \\
\hline Figure 9c & 214.0 & 75.7 \\
\hline Figure 9d & 214.0 & 75.7 \\
\hline Figure 9e & 216.8 & 75.4 \\
\hline
\end{tabular}

We next examine both the homogenization and the enrichment techniques using 900 quadratic $2^{\text {nd }}$ order elements, again examining the strain energy of the model. Table 3 shows that the homogenization method using the damaged RVE does not show much improvement in the strain energy calculations, with less than a \% difference between the two simulations. Using the enrichment method without any damaged RVEs still reduced the error by $6 \%$ over the homogenization approaches. When using the enrichment method along with the damaged RVE reduced the error to $60.4 \%$, a $15 \%$ improvement over the homogenization method.

Table 3. Strain Energy Errors

\begin{tabular}{|c|c|c|}
\hline Technique & Strain Energy (MPa) & Error (\%) \\
\hline Fine Mesh & 882.4 & - \\
\hline $\begin{array}{c}\text { Homogenization w/o } \\
\text { Damaged RVEs }\end{array}$ & 213.1 & 75.8 \\
\hline $\begin{array}{c}\text { Homogenization with } \\
\text { Damaged RVEs }\end{array}$ & 216.8 & 75.4 \\
\hline $\begin{array}{c}\text { Enrichment w/o } \\
\text { Damaged RVEs }\end{array}$ & 273.5 & 69.0 \\
\hline Enrichment with \\
Damaged RVEs
\end{tabular}




\section{CONCLUSIONS}

In this paper we have derived the enrichment functions for microdamage in a heterogeneous medium. This method uses a structural based enrichment technique, allowing macroscale computations to be performed with the microstructural features explicitly considered. In section three we have demonstrated that using the enriched approach with damaged RVEs reduces the error, for the problem shown, by $15 \%$. We are continuing this research for dynamic problems and are expecting to compare the results with experimental data.

\section{REFERENCES}

1. Nemat-Nasser, S \& Hori, M. Micromechanics: Overall Properties of Heterogeneous Materials, NorthHolland, London, 1993.

2. Bakhalov, N. \& Panasenko, G. Homogenization: Averaging process in periodic media. Dordrecht: Kluer: Academic Publishers, 1989.

3. Fish, J. \& Yuan, Z. "Multiscale enrichment based on partition of unity." International Journal for Numerical Methods in Engineering 24 (2005): 1341-1359.

4. Macri, M. \& De, S. "An octree partition of unity method (OctPUM) with enrichments for multiscale modeling of heterogeneous media." Computers \& Structures 86 (2008): 780-795.

5. Macri, M. \& Littlefield A. “Enrichment Based Multi-scale Modeling of Composite Materials undergoing Thermo-Stress", International Journal for Numerical Methods in Engineering 93 (2013): 1147-1169. 